\title{
Application of Nano-Cellulose in Textile
}

\section{Salah Mnaour Salah*}

Profesor, Textile chemistry department, Cotton Research Institute, Cairo, Egypt

Nano cellulose Nanotechnology is a strongly increasing area of research, training and development, and one that holds great promise for sustainable applications. Cellulose is a material widespread in nature and it may be obtained from many natural sources such as wood, cotton, and vegetable biomass. Cellulose is the most abundant and renewable natural resource on earth. Suitable prepared cellulose in the form of powder (nanocellulose or microfiberillated cellulose) is very useful products. They have been applied in pharmaceutical, food, ceramics, paint, textile and many other industries. Such a wide application is attributed to their special properties: nano dimensional particle size, large specific areas, and internees to many organic or inorganic substances. Nano- cellulose derived from natural sources is classified as nano-products. Both the bottom-up and top-down applicable to the production of nano-cellulose. Nano-cellulose can also be obtained from native fibers by an acid hydrolysis, giving rise to highly crystalline and rigid nanoparticles (generally referred to as nanowhiskers) which are shorter (100s to 1000 nanometers) than the nanofibrils obtained through the homogenization route. The resulting material is known as nano-crystalline cellulose (NCC). Typical lateral dimensions are 5-20 nanometers and longitudinal dimension is in a wide range from tens of nanometers to several micrometers. It is pseudo-plastic and exhibits the property of certain gels or fluids that are thick (viscous) under normal conditions, but flow (become thin, less viscous) over time when shaken, agitated, or otherwise stressed. This property is known as thixotropy. Cellulosic materials intended for use as nano-fillers in composites are usually subjected to hydrolysis by strong acids such as sulfuric or hydrochloric acid, which causes a selective degradation of amorphous regions of cellulose and, consequently, the splitting of micro-fibril beams. As a result of cellulose hydrolysis, the disintegration of its hierarchical structure takes place to form crystalline nanofibers or nanocrystals, usually referred to in literature as nanowhiskers. The morphology of the nanocrystals obtained depends on the source of cellulose and hydrolysis conditions used, such as acid concentration, the ratio of acid to cellulose, temperature and reaction time. Application of engineered nanomaterials has offered a new generation of environmental in textile industries. Fibres used in the textile industry are notably improved by nanotechnology; one example is nanocellulose, which combines low cost, lightweight, electric conductivity, environmentally friendly resources and high resistance, thus opening an immensely vast scope of possible applications. Certain smart nanofabrics can clean themselves by taking full advantage of the lotus effect, or they could be UV blocking, flame retardant or may never wear out. In any case, they are likely to enter the very fabric of our lives, as they could eventually be used for wireless biomonitoring of vital functions at a distance. Antibacterial textiles with embedded nanoparticles are going to have a major impact in hospitals and care homes, where cross-contamination of bacteria can be dangerous, especially to the elderly and the immune-suppressed. Effective control of bacteria populations in those environments will lead to reduced infection rates.

The innovative potential of nanotechnologies offers a wide span of opportunities for the textile industry. With the help of nanotechnologies, textile materials can be improved for both professional and general public users; whether nano-engineered materials are integrated into the polymer matrix or coated onto the surface of the fibres, Cotton fibers are natural fibers composed of fibers in different length. Formation of microcrystalline cellulose from the short fibers forming from snap of long fiber during the spinning systems are made by acid hydrolysis. The produced powder was coated onto natural or synthetic fabrics. They offer possibilities such as improved durability, self-cleaning, and wateror dirt- repellent features.

Through the manufacture of smart- or e-textiles, nanotechnologyenabled apparel can both protect the wearer from pathogens, toxic gases and other hazardous substances, benefiting the medical and rescue services as well as in the military, and allow the constant monitoring of body functions in applications ranging from regenerative activities to the enhancement of the quality of life of sufferers of long-term diseases. In the field of agriculture and crop optimization, nanofibers that have the ability to absorb plant protection products are applied to achieve controlled slow release functionalities.
*Corresponding author: Salah Mnaour Salah, Profesor, Textile chemistry department, Cotton Research Institute, Cairo, Egypt, E-mail: salahmansour9042@hotmail.com

Received October 31, 2013; Accepted November 01, 2013; Published November 07, 2013

Citation: Salah SM (2013) Application of Nano-Cellulose in Textile. J Textile Sci Eng 3: 142. doi:10.4172/2165-8064.1000142

Copyright: (C) 2013 Salah SM. This is an open-access article distributed under the terms of the Creative Commons Attribution License, which permits unrestricted use, distribution, and reproduction in any medium, provided the original author and source are credited. 